\title{
ANOTHER VEECH TRIANGLE
}

\author{
W. PATRICK HOOPER
}

(Communicated by Michael Wolf)

\begin{abstract}
We show that the triangle with angles $\frac{\pi}{12}, \frac{\pi}{3}$, and $\frac{7 \pi}{12}$ has the lattice property and we compute this triangle's Veech group.
\end{abstract}

In a 1989 paper of Veech, a property of a polygon $P$ in the plane was found which implies that the number of combinatorially distinct periodic billiard paths of length less than $t, N(P, t)$, satisfies

$$
\lim _{t \rightarrow \infty} \frac{1}{t^{2}} N(P, t)=c(P),
$$

where $c(P)$ is a constant depending only on the polygon Vee89. This property is now known as the lattice property. Since that time, the question of which polygons have the lattice property has been studied. The goal of this paper is to demonstrate that the triangle $\Delta$ with angles $\left(\frac{\pi}{12}, \frac{\pi}{3}, \frac{7 \pi}{12}\right)$ has Veech's lattice property.

The Zemljakov-Katok construction associates a polygon $P$ with a surface $S_{P}$ [ZK75]. To construct $S_{P}$, we define a subgroup $G$ of the orthogonal group $O(2)$ generated by reflections in the sides of $P$. Then we define

$$
S_{P}=\bigsqcup_{g \in G} g(P) / \sim,
$$

where $\sim$ is an equivalence relation defined by gluing edges. For every edge $e \subset P$, we glue $g_{1}(P)$ and $g_{2}(P)$ by an orientation-reversing isometry along the edges $g_{1}(e)$ and $g_{2}(e)$ whenever $g_{1} g_{2}^{-1}$ is the element of $O(2)$ obtained by reflection in the side $e$ of $P$. These edge identifications glue edges of polygons by translations. Assuming the group $G$ is finite, the surface $S_{P}$ has finite area. Figure 1 shows the triangle $\Delta$ together with the surface $S_{\Delta}$.

The surface $S_{\Delta}$ fits into an infinite class of surfaces with the lattice property of genus 4 discovered by McMullen McM06. See the next section for further historical details.

The surface $S_{P}$ is a translation surface, a surface built from polygons in $\mathbb{R}^{2}$ with edges glued by translations. Translation surfaces typically have singular points which are cone points with cone angles that are integer multiples of $2 \pi$. An affine automorphism of a translation surface $S$ is a homeomorphism $\phi: S \rightarrow S$ which

Received by the editors July 25, 2011.

2010 Mathematics Subject Classification. Primary 14H37.

The author was supported by N.S.F. Postdoctoral Fellowship DMS-0803013.

(C)2012 American Mathematical Society Reverts to public domain 28 years from publication 


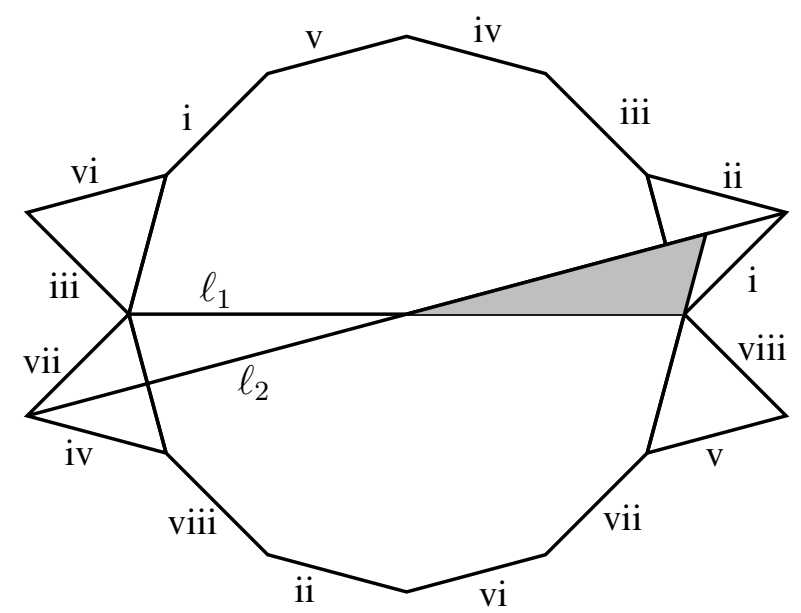

Figure 1 . The triangle $\Delta$ together with the corresponding translation surface, $S_{\Delta}$. Roman numerals indicate edge identifications.

preserves the underlying affine structure. By identifying tangent planes of nonsingular points with the plane, we see that an affine automorphism has a welldefined derivative $D(\phi): \mathbb{R}^{2} \rightarrow \mathbb{R}^{2}$ and that

$$
D(\phi) \in \widehat{S L}(2, \mathbb{R})=\{M \in G L(2, \mathbb{R}) \mid \operatorname{Det}(M)= \pm 1\} .
$$

The Veech group $\Gamma(S) \subset \widehat{S L}(2, \mathbb{R})$ of a translation surface $S$ is the group of derivatives of affine automorphisms of $S$. The surface $S$ has the lattice property if $\mathbb{H}^{2} / \Gamma(S)$ has finite hyperbolic area.

In this paper, we prove the following theorem.

Theorem 1. The surface $S_{\Delta}$ has the lattice property. A fundamental domain for the action of the affine automorphism group $\Gamma\left(S_{\Delta}\right) \subset \widehat{S L}(2, \mathbb{R})$ on the hyperbolic plane is shown in Figure 2. $\Gamma\left(S_{\Delta}\right)$ is generated by reflections in the sides of this polygon together with $-I$.

\section{Historical REMARKS}

Theorem 1 adds an additional triangle to the list of known triangles with the lattice property. This list follows.

(1) The acute isosceles triangles with angles $\left(\frac{(n-1) \pi}{2 n}, \frac{(n-1) \pi}{2 n}, \frac{\pi}{n}\right)$ for $n \geq 3$ (due to Veech Vee89]).

(2) The acute triangles $\left(\frac{\pi}{4}, \frac{\pi}{3}, \frac{5 \pi}{12}\right),\left(\frac{\pi}{5}, \frac{\pi}{3}, \frac{7 \pi}{15}\right),\left(\frac{2 \pi}{9}, \frac{\pi}{3}, \frac{4 \pi}{9}\right)$ (due to Veech Vee89, Vorobets [Vor96, and Kenyon and Smillie [KS00] respectively).

(3) The right triangles with angles $\left(\frac{\pi}{n}, \frac{(n-2) \pi}{2 n}, \frac{\pi}{2}\right.$ ) for $n \geq 4$ (due to Veech Vee89]).

(4) The obtuse isosceles triangles with angles $\left(\frac{\pi}{n}, \frac{\pi}{n}, \frac{(n-2) \pi}{n}\right)$ with $n \geq 5$ (due to Veech [Vee89]).

(5) The obtuse triangles with angles $\left(\frac{\pi}{2 n}, \frac{\pi}{n}, \frac{(2 n-3) \pi}{2 n}\right.$ ) with $n \geq 4$ (due to Ward War98). 


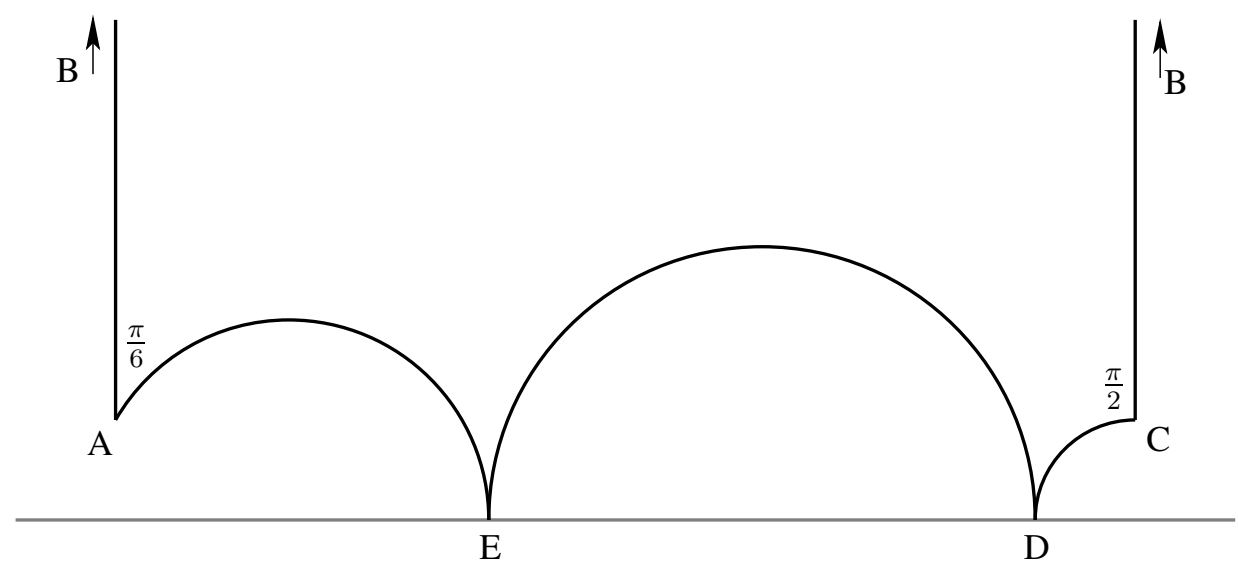

Figure 2. The fundamental domain for the action of $\Gamma\left(S_{\Delta}\right)$ on the upper half-plane is the polygon pictured. The polygon is the hyperbolic convex hull of its vertices: $A=i, B=\infty, C=5+$ $3 \sqrt{3}+i, D=4+3 \sqrt{3}$, and $E=2+\sqrt{3}$.

The work of Kenyon and Smillie KS00 together with that of Puchta Puc01 has shown that this list is complete in the case of acute, right, and isosceles triangles. Kenyon and Smillie provided a simple criterion which can be used to decide that a given triangle does not have the lattice property. This criterion requires knowledge of the existence of a periodic billiard trajectory. Kenyon and Smillie used the Fagnano curve, which is a periodic billiard path in every acute triangle, to rule out all but a short list of acute triangles with angles that are rational multiples of $\pi$ with denominator less than 10,001. Puchta later eliminated the remaining acute triangles.

The triangle $\Delta$ with angles $\left(\frac{\pi}{12}, \frac{\pi}{3}, \frac{7 \pi}{12}\right)$ was found using the methods of Kenyon and Smillie. Rich Schwartz and the author have written a computer program called McBilliard 11 which is capable of finding periodic billiard trajectories in triangles. This program together with Kenyon and Smillie's criterion enabled the author to (non-rigorously) search for triangles which might satisfy the lattice property. The triangle $\Delta$ was the only new triangle with angles of small denominator which seemed to pass this test. (The test did not check for certain numerical errors.) This suggests that the answer to the following question is likely to be "yes".

Open Question 2. Is the list of obtuse triangles with the lattice property now complete?

This triangle was found by the author in 2005, and the result was included in the author's Ph.D. thesis Hoo06. As this surface was discovered by the author, McMullen was finishing work on the paper [McM06], which provides infinite lists of surfaces with the lattice property in genera 3 and 4 . McMullen was able to check that the surface $S_{\Delta}$ fit into his infinite list. See also the remarks in [McM06] at the end of section 1.

Thus there are two proofs that $S_{\Delta}$ has the lattice property. In [McM06, McMullen gives a proof which does not explicitly yield the Veech group but works

\footnotetext{
${ }^{1}$ McBilliards is freely available from http://mcbilliards.sourceforge.net/.
} 
for a more general class of surfaces. Our main motivation is to explicitly compute the Veech group. We can see from this computation that the triangle $\Delta$ is special: it is the only triangle with the lattice property that has a Veech group which is not a triangle group.

\section{Outline}

In the following section, we will define the affine automorphism group and give enough background to prove the theorem. The theorem's proof lies in section 4 . In the final section, we will describe another affinely equivalent translation surface with Euclidean symmetry group of order 8 . This alternate picture of the surface realizes $S_{\Delta}$ as one of McMullen's X-shaped polygons. See McM06.

\section{BACKGROUND}

In this section, we briefly define a translation surface, its affine automorphism group, and the lattice property. For more details, see MT02].

A translation surface is a closed oriented surface $S$ together with a discrete set $\Sigma \subset S$ and an atlas of charts from $S \backslash \Sigma$ to the plane so that the transition functions are translations. The subset $\Sigma$ is known as the singular set. The atlas of charts is a covering of $S \backslash \Sigma$ by open sets $U_{i}$ together with local homeomorphisms $\phi_{i}: U_{i} \rightarrow \mathbb{R}^{2}$. The transition functions are the maps $\phi_{i} \circ \phi_{j}^{-1}$ restricted to $\phi_{j}\left(U_{i} \cap U_{j}\right)$. The translation surface inherits the pull-back metric from the plane and also the notion of direction. Small open sets of $S \backslash \Sigma$ are thus isometric to the plane and the points of $\Sigma$ are cone points that have cone angles which are integer multiples of $2 \pi$. The relevant example of a translation surface is shown in Figure 1

We will let $\widehat{S L}(2, \mathbb{R})$ be the subgroup of affine transformations of the plane that preserve area and fix the origin. See equation (2). An element $A \in \widehat{S L}(2, \mathbb{R})$ acts affinely on the plane. Given $S$ we can form a new translation surface $A(S)$ by postcomposing the charts of $S$ with $A$. The transition functions of $A(S)$ are translations, since they are just the transition functions of $S$ conjugated by $A$. Thus, $A(S)$ is another translation surface.

The affine automorphism group, $\Gamma(S) \subset \widehat{S L}(2, \mathbb{R})$, of $S$ is the set of elements $A \in$ $\widehat{S L}(2, \mathbb{R})$ so that there is a direction-preserving isometry $\phi: S \rightarrow A(S)$. (Directionpreserving is important; otherwise rotations would automatically be in $\Gamma(S)$.) A translation surface $S$ is said to have the lattice property if $\Gamma(S) \subset \widehat{S L}(2, \mathbb{R})$ is a lattice.

Veech discovered a relevant and powerful lemma about parabolics in the affine automorphism group in terms of cylinders of the surface. See Vee89] and [MT02. The modulus of a cylinder is the height of the cylinder divided by its circumference.

Lemma 3 (Veech). There is a parabolic in the group $\Gamma(S)$ fixing the direction $\theta$ if and only if there is a decomposition of the surface into cylinders in the direction $\theta$ whose moduli are commensurable (rational multiples of one another).

The following makes this lemma more explicit.

Discussion 4. Suppose $\theta$ is the horizontal direction and $\alpha$ is the greatest common divisor of the moduli of the cylinders in the horizontal decomposition given by the lemma. The greatest common divisor of a set of commensurable numbers 
$\left\{m_{1}, \ldots, m_{n}\right\}$ is the largest number $\alpha$ so that $\frac{m_{i}}{\alpha} \in \mathbb{Z}$ for all $i$. The generating parabolic fixing $\theta$ is given by

$$
\left(\begin{array}{ll}
1 & \frac{1}{\alpha} \\
0 & 1
\end{array}\right)
$$

To aid in visualizing $\Gamma(S)$, it is worth considering the action of $\widehat{S L}(2, \mathbb{R})$ on the upper half-plane. The group $\widehat{S L}(2, \mathbb{R})$ of equation (2) acts on the upper half-plane by hyperbolic isometries in the standard way. The upper half-plane is a subset of the Riemann sphere, $\hat{\mathbb{C}}=\mathbb{C}^{2} /(\mathbb{C} \backslash\{0\})$. The upper half-plane is the equivalence classes of elements $(z, 1) \in \mathbb{C}^{2}$ where $z$ has positive imaginary part. An element of $\widehat{S L}(2, \mathbb{R})$ acts on $\hat{\mathbb{C}}$ as follows:

$$
\left(\begin{array}{ll}
a & b \\
c & d
\end{array}\right)\left(\begin{array}{l}
z \\
w
\end{array}\right)=\left\{\begin{array}{l}
\left(\begin{array}{l}
a z+b w \\
c z+d w \\
a \bar{z}+b \bar{w} \\
c \bar{z}+d \bar{w}
\end{array}\right) \quad \text { if } a d-b c=1 \\
\text { if } a d-b c=-1
\end{array}\right.
$$

Note that this action is not faithful because $-I$ acts trivially.

\section{THE PROOF}

We break up the proof of the theorem into two lemmas. In the first we prove that the elements we list are in $\Gamma\left(S_{\Delta}\right)$. Then we will show that this list generates all of $\Gamma\left(S_{\Delta}\right)$.

Lemma 5. Each of the reflections in the side of the polygon of Figure 2 is in $\Gamma\left(S_{\Delta}\right) .-I$ is also in $\Gamma\left(S_{\Delta}\right)$.

Proof. The surface $S_{\Delta}$ has several Euclidean automorphisms. The element $-I$ acts on the plane by a Euclidean rotation by $\pi$. Thus, it is clear that $-I \in \Gamma\left(S_{\Delta}\right)$. The Euclidean automorphism group is generated by reflections in lines $\ell_{1}$ and $\ell_{2}$ of Figure 1. This gives two of our generators:

$$
R_{\overline{A B}}=\left(\begin{array}{cc}
1 & 0 \\
0 & -1
\end{array}\right) \quad \text { and } \quad R_{\overline{A E}}=\left(\begin{array}{cc}
\frac{\sqrt{3}}{2} & \frac{1}{2} \\
\frac{1}{2} & -\frac{\sqrt{3}}{2}
\end{array}\right) .
$$

Now we will find a parabolic automorphism of $S_{\Delta}$ fixing the point $B$. There is a decomposition of $S_{\Delta}$ by saddle connections parallel to $\ell_{1}$ of Figure 1 . This decomposition is depicted in Figure 3 and cuts the surface into 4 cylinders. It can be verified that these cylinders come in pairs with two possible moduli:

$$
\frac{1}{5+3 \sqrt{3}} \text { and } \frac{1}{10+6 \sqrt{3}} \text {. }
$$

In particular, note that the first modulus is twice the second. Thus, there is a parabolic element of the automorphism group which fixes the horizontal direction and acts as a single Dehn twist on the pair of cylinders with modulus $\frac{1}{10+6 \sqrt{3}}$ and a double Dehn twist on the pair of cylinders with modulus $\frac{1}{5+3 \sqrt{3}}$. This parabolic is:

$$
P_{B}=\left(\begin{array}{cc}
1 & 10+6 \sqrt{3} \\
0 & 1
\end{array}\right)
$$



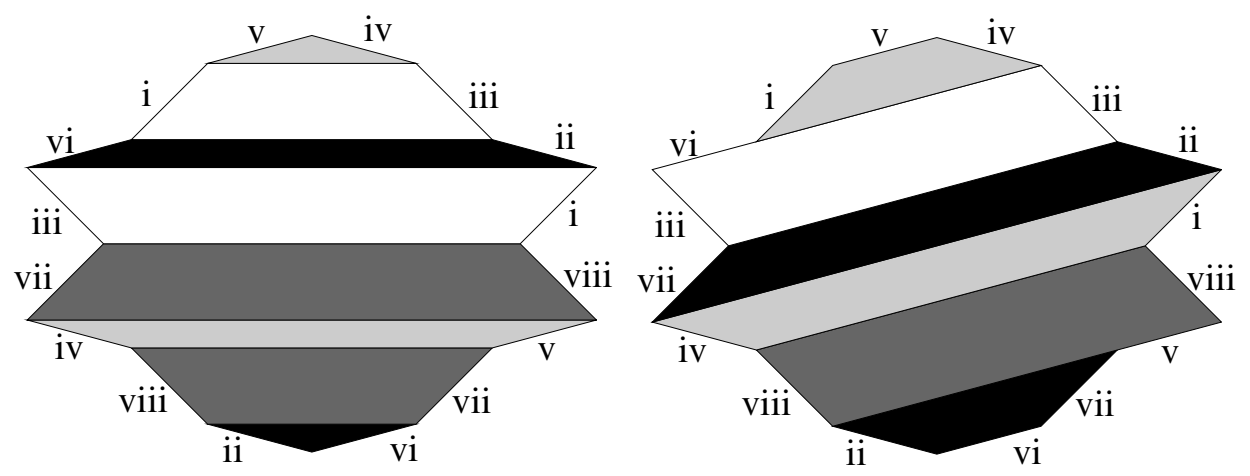

Figure 3. Decompositions into cylinders using saddle connections parallel to lines $\ell_{1}$ and $\ell_{2}$ of Figure 1 .

Both the reflection $R_{\overline{A B}}$ and the parabolic $P_{B}$ fix the point $B$, so their composition does as well. Their composition gives another reflection:

$$
R_{\overline{B C}}=P_{B} \circ R_{\overline{A B}}=\left(\begin{array}{cc}
1 & -10-6 \sqrt{3} \\
0 & -1
\end{array}\right) .
$$

The same idea will work for generating a reflection preserving points $E$ and $D$. We decompose $S_{\Delta}$ into 4 cylinders using segments parallel to $\ell_{2}$ of Figure 1 This decomposition is shown on the right in Figure 3 . The moduli of these cylinders again come in pairs:

$$
\frac{3}{6+4 \sqrt{3}} \text { and } \frac{1}{6+4 \sqrt{3}}
$$

This means that there is a parabolic inducing a single Dehn twist on the cylinders with moduli $\frac{1}{6+4 \sqrt{3}}$ and a triple Dehn twist on the other cylinders. This parabolic is

$$
P_{E}=\left(\begin{array}{cc}
\frac{1}{2}(-1-2 \sqrt{3}) & \frac{1}{2}(12+7 \sqrt{3}) \\
\frac{1}{2}(-\sqrt{3}) & \frac{1}{2}(5+2 \sqrt{3})
\end{array}\right) .
$$

Again we obtain $R_{\overline{D E}}$ by composition:

$$
R_{\overline{D E}}=R_{\overline{A E}} \circ P_{E}=\left(\begin{array}{cc}
\frac{1}{2}(-3-\sqrt{3}) & \frac{1}{2}(13+7 \sqrt{3}) \\
\frac{1}{2}(1-\sqrt{3}) & \frac{1}{2}(3+\sqrt{3})
\end{array}\right) .
$$

We apply the same trick one last time. $R_{\overline{D E}}$ preserves two parallel families of lines on the surface, each corresponding to eigenvectors of the matrix. Of course, one is the family of lines parallel to $\ell_{2}$. The second family has slope $\frac{1}{11}(-4+3 \sqrt{3})$. We decompose the surface using saddle connections parallel to this direction (see Figure 4). Again, these cut the surface into four cylinders whose moduli come in pairs. The moduli are

$$
\frac{1}{29+17 \sqrt{3}} \text { and } \frac{1}{58+34 \sqrt{3}} \text {. }
$$

Therefore, we get a parabolic fixing lines of slope $\frac{1}{11}(-4+3 \sqrt{3})$ :

$$
P_{D}=\left(\begin{array}{cc}
\frac{1}{2}(-11-7 \sqrt{3}) & \frac{1}{2}(115+67 \sqrt{3}) \\
\frac{1}{2}(-1-\sqrt{3}) & \frac{1}{2}(15+7 \sqrt{3})
\end{array}\right) .
$$




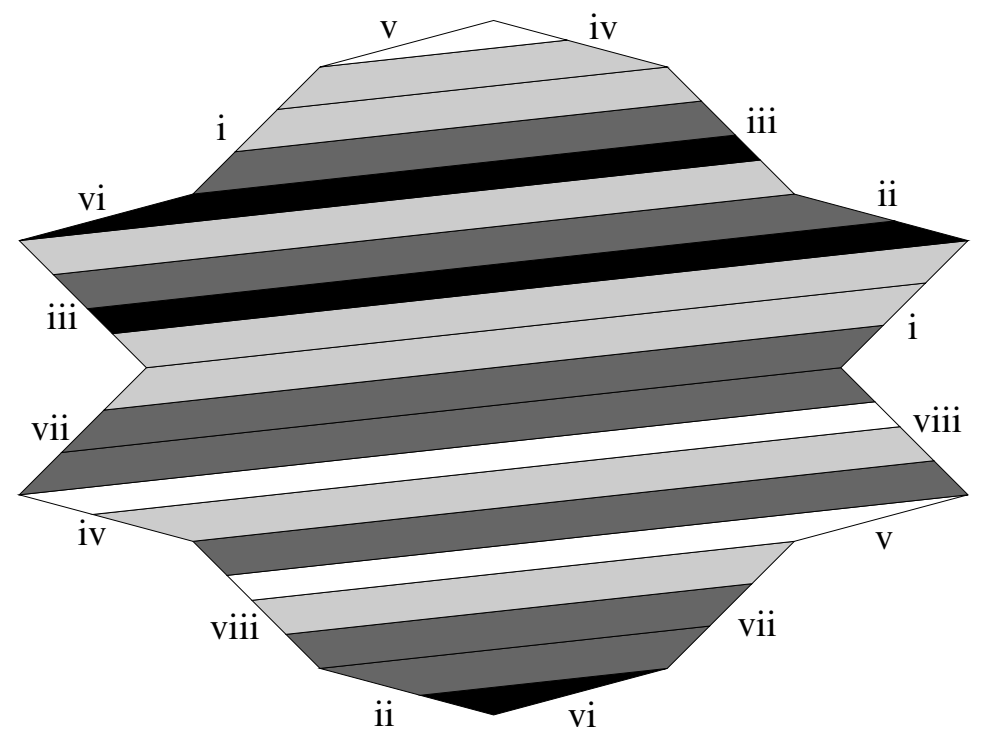

FIgURE 4. Saddle connections with slope $\frac{1}{11}(-4+3 \sqrt{3})$ decompose the surface into these cylinders.

We compose the parabolic with the reflection $R_{\overline{D E}}$ :

$$
R_{\overline{C D}}=R_{\overline{D E}} \circ P_{D}=\left(\begin{array}{cc}
5+3 \sqrt{3} & -51-30 \sqrt{3} \\
1 & -5-3 \sqrt{3}
\end{array}\right) .
$$

One way to check that lines $\overline{B C}$ and $\overline{C D}$ intersect at a right angle is to compute the trace of the product of reflections in the sides. If this trace is zero, then the sides meet at a right angle. We compute

$$
\operatorname{Tr}\left(R_{\overline{B C}} \circ R_{\overline{C D}}\right)=\operatorname{Tr}\left(\begin{array}{cc}
-5-3 \sqrt{3} & 53+30 \sqrt{3} \\
-1 & 5+3 \sqrt{3}
\end{array}\right)=0 .
$$

Lemma 6. The reflections in the side of the polygon of Figure 2 together with $-I$ generate the affine automorphism group $\Gamma\left(S_{\Delta}\right)$.

Proof. Let $G$ be the group generated by $-I$ and the reflections in the sides of the polygon of Figure 2, and let $G^{+}$be the index 2 subgroup which preserves the orientation of $\mathbb{H}^{2}$.

Let $Y_{G}=\mathbb{H}^{2} / G^{+}$, a sphere with 3 punctures and two cone singularities. One singularity has cone angle $\pi$ and the other has cone angle $\pi / 3$. We can compute the area of $Y_{G}$ using the Gauss-Bonnet formula. Recall that the Gauss-Bonnet formula for hyperbolic surfaces with cone singularities tells us that for a surface $S$ of genus $g$ with $p$ punctures and cone singularities of cone angles $\theta_{1}, \ldots, \theta_{n}$,

$$
\operatorname{area}(S)=2 \pi(2 g+p-2)+\sum_{i=1}^{n}\left(2 \pi-\theta_{i}\right)
$$

We compute that $\operatorname{area}\left(Y_{G}\right)=\frac{14 \pi}{3}$. 
Now let $V=\Gamma\left(S_{\Delta}\right)$ be the complete affine automorphism group, $V^{+}$be the orientation-preserving subgroup, and $Y_{V}=\mathbb{H}^{2} / V^{+}$. We wish to show that $V=G$. The previous lemma showed that $G$ is a subgroup of $V$. Thus we have a covering map $\psi: Y_{G} \rightarrow Y_{V}$. Further we know that

$$
\left[V^{+}: G^{+}\right]=\operatorname{area}\left(Y_{G}\right) / \operatorname{area}\left(Y_{V}\right),
$$

where $\left[V^{+}: G^{+}\right]$is the index of the subgroup $G^{+}$inside $V^{+}$. In order to show that $V^{+}=G^{+}$, it is sufficient to show that area $\left(Y_{G}\right) / \operatorname{area}\left(Y_{V}\right)<2$.

We would like to use Gauss-Bonnet on $Y_{V}$. First we will show that $Y_{V}$ also has 3 punctures. It is sufficient to show that none of the punctures of $Y_{G}$ can be identified by $\psi$. We will give affine invariants which distinguish the three decompositions into cylinders mentioned in the previous proof. The ratio of the moduli of the cylinders associated to the vertex $E$ of the polygons is 3, while the ratios of the moduli of cylinders associated to $B$ and $D$ are both 2 (see equations (8), (5), and (11)). Thus $E$ cannot be identified with $B$ or $D$. Another affine invariant is the ratio of the widths of the cylinders. We can compute that these ratios are

$$
w_{B}=1+\sqrt{3} \text { and } w_{D}=\frac{1+\sqrt{3}}{2} .
$$

Thus, the punctures coming from $B$ and $D$ cannot be identified by the covering $\psi$. This shows that $Y_{V}$ has 3 punctures.

We also need to show that $Y_{V}$ has at least one cone singularity. The image of a cone singularity in $Y_{G}$ must be a cone singularity in $Y_{V}$. Further, the image of the cone singularity with cone angle $\pi / 3$ must be a cone singularity with cone angle $\theta$ which is less than $\pi / 3$. Gauss-Bonnet now tells us that

$$
\operatorname{area}\left(Y_{V}\right) \geq 2 \pi(3-2)+(2 \pi-\theta) \geq 4 \pi-\pi / 3=11 \pi / 3>\frac{1}{2} \operatorname{area}\left(Y_{G}\right) .
$$

Thus $\operatorname{area}\left(Y_{G}\right) / \operatorname{area}\left(Y_{V}\right)<2$, so $\left[V^{+}: G^{+}\right]=1$ and $V^{+}=G^{+}$.

Finally, because both $V$ and $G$ contain orientation-reversing elements, we know that $[V: G]=\left[V^{+}: G^{+}\right]$. Thus $V=G$.

\section{The Other Symmetric translation SURFACE}

The translation surface $S_{\Delta}$ is affinely equivalent to another translation surface $S^{\prime}$. This surface corresponds to the vertex $C$ of the fundamental domain in $\mathbb{H}^{2}$ of the affine automorphism group of $S_{\Delta}$ pictured in Figure2 Thus, $S^{\prime}$ can be written as $A\left(S_{\Delta}\right)$, where $A \in \widehat{S L}(2, \mathbb{R})$ is

$$
A=\left(\begin{array}{cc}
1 & 5+3 \sqrt{3} \\
0 & 1
\end{array}\right) .
$$

The surface $S^{\prime}$ is pictured in Figure 5 and supports a Euclidean isometry group $G$, which is a dihedral group of order 8 . The quotient $S^{\prime} / G$ is an annulus, which we will now describe. Consider the points

$$
\begin{gathered}
P_{0}=(0,0), \quad P_{1}=(1,0), \quad P_{2}=\left(\frac{3+\sqrt{3}}{4}, \frac{-1+\sqrt{3}}{4}\right), \\
P_{3}=\left(\frac{1+\sqrt{3}}{4}, \frac{1+\sqrt{3}}{4}\right), \quad P_{4}=\left(\frac{1}{2}, \frac{1}{2}\right) .
\end{gathered}
$$

$S^{\prime} / G$ is the pentagon $P_{0} P_{1} P_{2} P_{3} P_{4}$ with edge $\overline{P_{1} P_{2}}$ glued to $\overline{P_{4} P_{3}}$ by translation. 


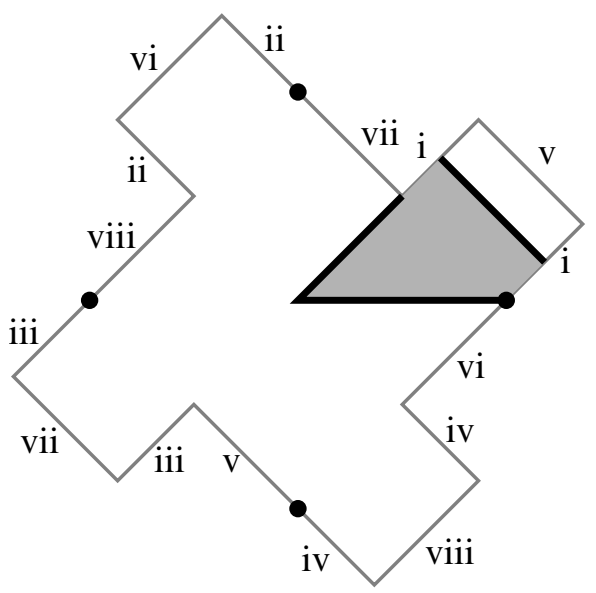

Figure 5. The translation surface $S_{\Delta}$ is affinely equivalent to this translation surface, $S^{\prime}$. The grey region is an annulus which arises as the quotient of this surface by its Euclidean isometries.

\section{ACKNOWLEDGEMEnts}

The author would like to thank Barak Weiss for the idea of searching for obtuse Veech triangles, and Curt McMullen for his encouragement to write this result down. The author would also like to thank Rich Schwartz for his collaboration on McBilliards and my graduate advisor, Yair Minsky.

\section{REFERENCES}

[Hoo06] W. Patrick Hooper, On the stability of periodic billiard paths in triangles, Ph.D. thesis, SUNY Stony Brook, Stony Brook, New York, 2006.

[KS00] Richard Kenyon and John Smillie, Billiards on rational-angled triangles, Comment. Math. Helv. 75 (2000), no. 1, 65-108. MR.1760496 (2001e:37046)

[McM06] Curtis T. McMullen, Prym varieties and Teichmüller curves, Duke Math. J. 133 (2006), no. 3, 569-590. MR2228463(2007a:32018)

[MT02] Howard Masur and Serge Tabachnikov, Rational billiards and flat structures, Handbook of dynamical systems, Vol. 1A, North-Holland, Amsterdam, 2002, pp. 1015-1089. MR.1928530 (2003j:37002)

[Puc01] Jan-Christoph Puchta, On triangular billiards, Comment. Math. Helv. 76 (2001), no. 3, 501-505. MR 1854695 (2002f:37060)

[Vee89] W. A. Veech, Teichmüller curves in moduli space, Eisenstein series and an application to triangular billiards, Invent. Math. 97 (1989), no. 3, 553-583. MR.1005006 (91h:58083a)

[Vor96] Ya. B. Vorobets, Plane structures and billiards in rational polygons: the Veech alternative, Uspekhi Mat. Nauk 51 (1996), no. 5(311), 3-42. MR1436653 (97j:58092)

[War98] Clayton C. Ward, Calculation of Fuchsian groups associated to billiards in a rational triangle, Ergodic Theory Dynam. Systems 18 (1998), no. 4, 1019-1042. MR.1645350 (2000b:30065)

[ZK75] A. N. Zemljakov and A. B. Katok, Topological transitivity of billiards in polygons, Mat. Zametki 18 (1975), no. 2, 291-300. MR0399423 (53:3267)

The City College of New York, New York, New York 10031

E-mail address: whooper@ccny.cuny.edu 